\title{
Influence of Coolant Concentration on Surface Roughness during Turning of Steel C-60
}

\author{
Abdulaziz Abodena, Muhannad Alrzage, Ibrahim K. Husain \\ Faculty of Engineering, University of Gharyan, University, Libya
}

DOI: https://doi.org/10.21467/proceedings.4.11

* Corresponding author email: abodena65@gmail.com

\begin{abstract}
During recent years, considerable attention has been paid to the during turning operations, to improve forming the outer layer, such as surface cooling which enhances some of the surface characteristics and producing a good surface finish. In this experimental work, the effect of cutting fluid concentrations on surface roughness has been studied because of its importance in the cutting process. The general aim of the work in this paper was to investigate the surface roughness of the workpiece when machining high carbon steel with tungsten inserts during wet machining conditions. In the experimentation, five different velocities, five feeds, five depths of cut and five coolant-oil content percentages were used to evaluate the surface roughness with these parameters. Design of experiments is a very efficient method for developing valuable research while saving time in the process. The technique used in this study for designing experiments was introduced by Box and Hunter. A rotatable factorial design of experiments with a central composite of second order can be used to enhance the reliability of investigation work. The package of design of experiments and analysis of variance used in this study has been used widely in experimentation work. The tests were performed using a traditional lathe machine. The results of this process were obtained, analyzed, and discussed using three-dimensional relationships between roughness and the cutting parameters mentioned above. The conclusions from this work show a general trend for a decrease in surface roughness as the percentage of oil in the coolant increases.
\end{abstract}

Keywords: Turning, surface roughness, cutting fluids.

\section{Introduction}

The nature of the surface region generated in metal machining may be quite different from that of bulk material. The surface may contain topographical features such as surface roughness. The subsurface may contain other features in the form of residual stress, plastic deformation and variation in microstructural characteristics such as hardening tempering over tempering, chemical composition and grain size [1]. Metal cutting is one of the vital processes and widely used manufacturing processes in engineering industries. Highly competitive market

(C) 2018 Copyright held by the author(s). Published by AIJR Publisher in Proceedings of First Conference for Engineering Sciences and Technology (CEST-2018), September 25-27, 2018, vol. 2.

This is an open access article under Creative Commons Attribution-NonCommercial 4.0 International (CC BY-NC 4.0)

license, which permits any non-commercial use, distribution, adaptation, and reproduction in any medium, as long as the original work is properly cited. ISBN: 978-81-936820-6-7 
Influence of Coolant Concentration on Surface Roughness during Turning of Steel C-60

requires high quality products at minimum cost. Improvement of productivity with good quality of the machined parts is the main challenges of metal industry; there has been more concern about monitoring all aspects of the machining process. Surface finish is an important parameter in manufacturing engineering and it can influence the performance of mechanical parts and the production costs[2]. During recent years, considerable attention has been paid to the turning operations, to improves forming the outer layer, such as surface cooling which enhances some of the surface characteristics and producing a good surface finish[1]. Cooling and lubrication are important in reducing the severity of the contact processes at the cutting tool-workpiece interfaces. Historically, more than 100 years ago, water was used mainly as a coolant due to its high thermal capacity and availability. Corrosion of parts and machines and poor lubrication were the drawbacks of such a coolant. Oils were also used at this time as these have much higher lubricity, but the lower cooling ability and high costs restricted this use to low cutting speed machining operations. Finally, it was found that oil added to the water gives good lubrication properties with the good cooling and these became known as the soluble oils[3, 4]. The main objective of this work is to study the effect of coolant concentration on the surface roughness of Carbon-Steel (C60) during the turning process, with other operating variables such as cutting speed, feed rate, and cutting depth, to determine the most suitable operating conditions to achieve the best surface smoothness. Even when considering the current trend towards research into minimum quantity lubrication processes, in many cases a flood of liquid is still directed over the tool with the aim of preventing the tool and workpiece from overheating, increasing tool life, and improving surface finish [5]. Selecting a suitable fluid for a particular application among the large number of commercially available fluids is an issue, and a significant challenge due to the fact it is often an empirical process.

\section{Experimental work}

In this study carbon steel alloy C60 was used as workpiece material. The chemical analyses, in weight percent, of this material is shown in Table (1). The material was received in the form of cylindrical bars with diameter of $60 \mathrm{~mm}$ and length of $70 \mathrm{~mm}$.

Table 2: Chemical composition of the C60 [6]

\begin{tabular}{|c|c|c|c|c|c|}
\hline Element & $\mathrm{Si}$ & Mo & $\mathrm{Cu}$ & $\mathrm{Mn}$ & $\mathrm{Ni}$ \\
\hline Weight $\%$ & max. 0.40 & max. 0.10 & 0.61 & 0.75 & max. 0.40 \\
\hline
\end{tabular}

\subsection{Cutting Conditions}

In this work, all of cutting tests are performed under lubricated conditions on (Al-pin 180N) type model of the lathe machine. In order to study in depth the effects of each parameter on the surface characteristics of workpiece, four cutting parameters were chosen namely; oil concentration, cutting speed, feed and cutting depth. The values of the actual and coded variables of the testing condition are listed in the following table 
Abodena et al., CEST-2018, AIJR Proceedings 4, pp.440-445, 2018

\begin{tabular}{|r|c|c|c|c|c|c||}
\hline \multicolumn{1}{|c|}{ Table 3: Coding of cutting test Parameters } \\
\hline \multirow{2}{*}{ Parameters } & \multirow{2}{*}{ Symbol } & \multicolumn{5}{c|}{ Levels } \\
\cline { 3 - 7 } & & -2 & -1 & 0 & 1 & 2 \\
\hline Coolant composition (\%) & $\mathrm{X}_{1}$ & 3 & 6 & 9 & 12 & 15 \\
\hline Speed, (m/min) & $\mathrm{X}_{2}$ & 56 & 120 & 150 & 412 & 840 \\
\hline Feed (mm/rev) & $\mathrm{X}_{3}$ & 0.055 & 0.09 & 0.18 & 0.3 & 0.4 \\
\hline Depth of cut (mm) & $\mathrm{X}_{4}$ & 0.2 & 0.6 & 1 & 1.4 & 1.8 \\
\hline
\end{tabular}

\subsection{Design of Experiments}

Traditional experimentation involves considerable effort and time, particularly when a wide range of investigation work is needed. Design of experiments is a very efficient method for developing valuable research while saving time in the process and for achieving results in a much more economical manner. As a rule, an experiment designed to find the optimum condition of a process is described adequately by a second order polynomial. The technique used in this study for designing experiments was introduced by box and Hunter.

Experimental work involves the study of the relationships between different factors at different levels and a certain response and this is where the design of experiments technique is helpful. There is a big opportunity to study the individual effects of each parameter and their interactions using the factorial design of experiments.

The package of design of experiments and analysis of variance used in this study has been used widely in experimentation work [7,8]. The spherical variance function designs are preferable since these designs provide a constant variance for the response at all points of the experiment since they are at the same radius from the centre of the design. These kinds of designs are known as rotatable designs.

\subsection{Postulation of Mathematical Model:}

A functional relationship between the response surface roughness of the workpiece produced by turning process in dependent variable (oil concentration, cutting speed, cutting feed and depth of cutting) can be fitted into the following polynomial response equation of secondorder.

$$
\begin{aligned}
& Y u=b_{o}+b_{1} x_{1}+b_{2} x_{2}+b_{3} x_{3}+b_{4} x_{4}+b_{11} x_{1}^{2}+b_{22} x_{2}^{2}+b_{33} x_{3}^{2}+b_{44} x_{4}^{2}+ \\
& b_{12} x_{1} x_{2}+b_{13} x_{1} x_{3}+b_{14} x_{1} x_{4}+b_{23} x_{2} x_{3}+b_{24} x_{2} x_{4}+b_{34} x_{3} x_{4}
\end{aligned}
$$

where; $x_{1}, x_{2}, x_{3}$ and $x_{4}$ are the coded values of the variables. These variables are coded for convenient identification and for easy calculation.

The regression coefficients $b_{o}, b_{1}, b_{3}, \ldots$ etc can be calculated by the method of least squares using the related equations. 
Influence of Coolant Concentration on Surface Roughness during Turning of Steel C-60

\subsection{Analysis of Variance (ANOVA):}

The relationship between the response and the cutting process parameters were quantitatively determined using empirical equations (the proposed model). The evaluation and the analysis of the experimental data were made by adopting polynomial response of second-order equations in terms of the process variables by establishing their interactions.

In the present work the variance for each of the regression coefficient is given by the related equations. The F-ratio for each term can then be determined from the variance analysis. In such an analysis, it is of interest to partition the sum of squares of the Y's into the contribution due to the first-order (Linear) term. An additional contribution due to the second-order (quadratic and interaction) terms, lack-of-fit terms (which measure the deviations of the response from the fitted surface), and the experimental error obtained from the replicated points at the center.

\section{Results and Discussion}

The model is developed in terms of Coolant concentration (\%) cutting speed, cutting depth and cutting feed by utilizing Response Surface Methodology. As it was mentioned above, the variables are investigated using the experimental design matrix instead of the conventional one-variable at a time method. The evaluation and the analysis of the experimental data is made by adopting a polynomial response surface of second-order in terms of the process variables by establishing their interaction. The results are tested statistically using the ANOVA technique presented previously. The F-ratio test as a tool of the analysis of variance is used to check the adequacy of the model. To determine whether the final equations are a good fit to the experimental observations, the F-ratio test is carried out. The standard valued of F-ratio for the significance level $\mathrm{a}=0.05$ and degrees of freedom 4 and 6 is F0.05(4,6) $=4.53$ and at degrees of freedom 10 and 6 is F0.05(10,6) $=4.06$.

$$
\begin{aligned}
\mathrm{Ra}=0.619+ & 0.708 \mathrm{x}_{1}-0.882 \mathrm{x}_{2}+0.98 \mathrm{x} 3-0.08 \mathrm{x} 4+2.14 \mathrm{x}_{1}{ }^{2}+2.06 \mathrm{x}_{2}{ }^{2} \\
& +0.317 \mathrm{x}_{3}{ }^{2}+0.32 \mathrm{x}_{4}{ }^{2}-0.42 \mathrm{x}_{1} \mathrm{x}_{2}+0.57 \mathrm{x}_{1} \mathrm{x}_{3}-0.11 \mathrm{x}_{1} \mathrm{x}_{4} \\
& -0.54 \mathrm{x}_{2} \mathrm{x}_{3}-0.38 \mathrm{x}_{2} \mathrm{x}_{4}+0.49 \mathrm{x}_{3} \mathrm{x}_{4}
\end{aligned}
$$

\subsection{Relation between roughness, oil content and speed}

Cutting speed and oil content are important parameters that effect on the roughness in cutting process. The figure (1) shows the result of the effect of oil content and speed on the roughness based on the empirical equation shown up. From the figure it is noticed that there is a change in the roughness value whenever the speed and the concentration of the oil change. It was noted that when the concentration of oil and speed is at the lowest levels, the roughness is the highest levels. When the concentration of oil increased to about $9 \%$ and the speed to about $150 \mathrm{~m} / \mathrm{min}$, it was noticed a decrease in the value of roughness until it reached its lowest value.

ISBN: 978-81-936820-6-7 


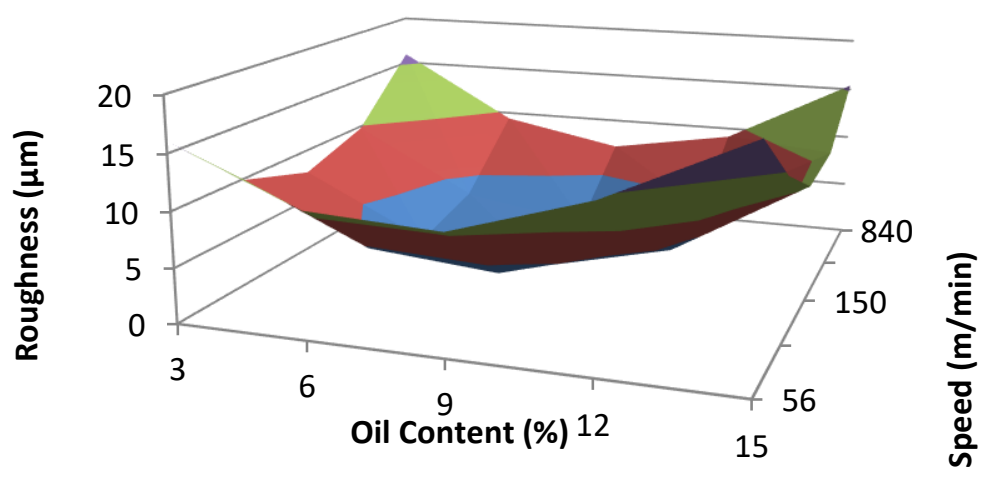

Figure 3: Relationship between roughness, oil content and cutting speed

\subsubsection{Effect of oil content and feed on roughness}

Figure (2) show the results of roughness versus different oil concentrations and cutting feed. The roughness is influenced by feed and oil concentration change. The best result of the number of smoothness is obtained at the minimum of the cutting feed and at concentration of oil about $9 \%$ used in this work.

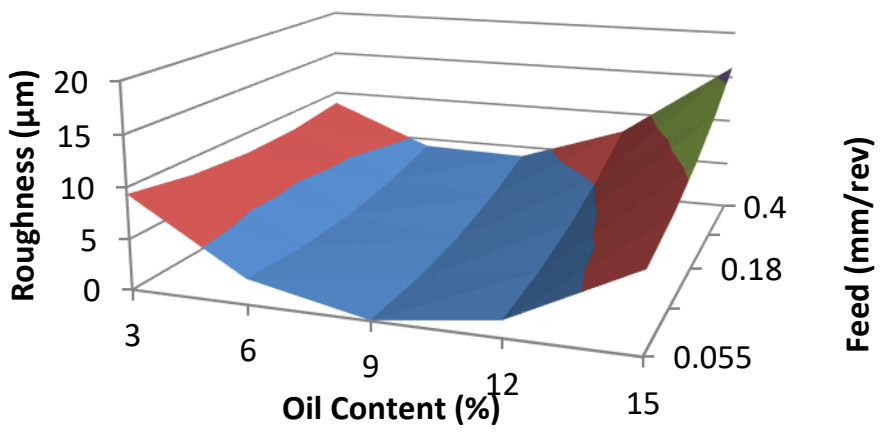

Figure 4: Relationship between roughness, oil content and cutting feed

\subsection{Relationship between roughness, oil content and depth}

Figure (3) shows the roughness versus different of oil content and depth of cut. The change in depth of cutting and oil content leads to change in roughness, low values of roughness is observed when oil concentrations are between $6 \%$ and $12 \%$ and the lowest value is at $9 \%$ and low depth of cut. 


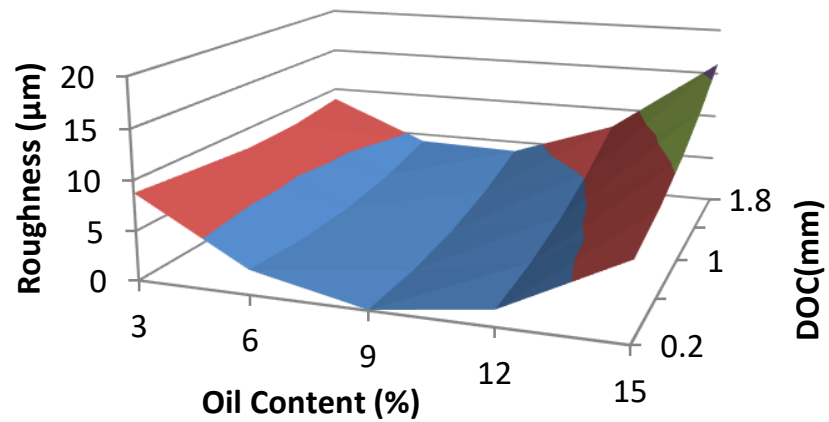

Figure 5: Relationship between roughness, oil content and depth of cutting

\section{Conclusions}

In this study 31 tests were made with different variables (oil concentration, cutting speed, cutting depth and feed) to reach the best value for surface smoothness. All these processes were applied to a group of samples of (C60). The following can be concluded; the results obtained from this work have shown that oil concentration has different effect on the response studied. An increase in oil concentration leads to improvement in surface finish up to a certain level. The recommended cutting feeds that result in good surface finish are from 0.055 to 0.18 $\mathrm{mm} / \mathrm{rev}$ when concentration of oil is $9 \%$. At speed of medium range $150 \mathrm{~m} / \mathrm{min}$ and concentration oil of 6 to $9 \%$, good surface finish can be obtained.

\section{References}

[1] EL-XEER, M.E.-K.M., EFFECT OF SOME BURNISHING PARAMETERS ON RESIDUAL STRESSES AND FATIGUE LIFE 2002.

[2] Bhavsara, H.Y.V.S.N., Experimental Investigation of Surface Roughness and Power Consumption in Turning Operation of EN 31 Alloy Steel. 2014: Elsevier Ltd.

[3] Machado, A., The effect of extremely low lubricant volumes in machining. 1997.

[4] McCoy, J., Tracing the historical development of metal workingfluids. 1994: New York: Marcel Dekker.

[5] Abodena, A.M. An Investigation into the Effect of Coolants' Concentration and Cutting Conditions on the Coefficient of Friction during Turning of Aluminum 6082-T6. in 1st Conference of Industrial Technology CIT2017. 2017. Misurata.

[6] Ravne., M. steel data. 2005; Available from: http://www.metalravne.com/selector/steels/C60.html.

[7] Joyce, R.M., Experiment optimization in chemistry and chemical engineering, S. Akhnazarova and V. Kafarov, Mir Publishers, Moscow and Chicago, 1982, 312 pp. Price: \$9.95. Journal of Polymer Science: Polymer Letters Edition, 1984. 22(6): p. 372-372.

[8] Allus, M.A., R.G. Brereton, and G. Nickless, The use of experimental design, multilinear regression, ANOVA, confidence bands and leverage in a study of the influence of metals on the growth of barley seedlings. Chemometrics and Intelligent Laboratory Systems, 1989. 6(1): p. 65-80. 